\title{
Galactic dynamo simulations
}

\section{Detlef Elstner, Oliver Gressel and Günther Rüdiger}

Astrophysikalisches Institut Potsdam, An der Sternwarte 16, D-14482 Potsdam, Germany email: elstner@aip.de

\begin{abstract}
Recent simulations of supernova-driven turbulence within the ISM support the existence of a large-scale dynamo. With a growth time of about two hundred million years, the dynamo is quite fast - in contradiction to many assertions in the literature. We here present details on the scaling of the dynamo effect within the simulations and discuss global meanfield models based on the adopted turbulence coefficients. The results are compared to global simulations of the magneto-rotational instability.
\end{abstract}

Keywords. ISM: magnetic fields - galaxies: magnetic fields

\section{Introduction}

Large-scale coherent magnetic fields are observed in many spiral galaxies (Beck et al. 1996; Beck 2009). It is now widely accepted that a turbulent dynamo process is responsible for the sustained amplification of the mean magnetic field within galaxies. The turbulence driven by supernova explosions together with the differential rotation should in fact lead to the action of the classical $\alpha \Omega$ dynamo (Parker 1971). First steps to globally model flat objects in an ellipsoidal geometry were done by Stix (1975); White (1978). Starting from the early nineties, first multidimensional numerical mean-field models assuming a disk geometry appeared (Donner \& Brandenburg 1990; Elstner et al. 1992). These models applied simple estimates for the turbulent transport coefficients in the mean-field induction equation.

Rüdiger \& Kitchatinov (1993), in the framework of second order correlation approximation (SOCA), derived the full $\alpha$ tensor and turbulent diffusivity for stratified turbulence. Fröhlich \& Schultz (1996), under the assumption of hydrostatic equilibrium, computed the turbulent velocity distribution $u^{\prime}(z)$ based on the observed density stratification and an observationally motivated gravitational potential from stars and dark matter. Applying SOCA theory, one could now specify the full $\alpha$ tensor, resulting in a model with the correlation time $\tau_{\mathrm{c}}$ of the turbulence as the only free parameter.

With the increased computer power of the nineties, Kaisig et al. (1993) and Ziegler (1996), in an alternative approach, computed the turbulence coefficients by averaging the results from simulations of a single supernova remnant. It, however, turned out that the values from isolated explosions were about one magnitude smaller then the estimated numbers from the previous considerations. Similar results, based on semi-analytical models, were obtained by Ferrière (1998). Extending the investigation to super-bubbles and including the stratification of the ISM, she finally found turbulence coefficients of expected magnitude. However, with a huge escape velocity, which could switch off the dynamo process. Ferrière and Schmitt (2000) used these results as input for an axisymmetric dynamo model of our Galaxy. They reached growth times of about $450 \mathrm{Myr}$ but with a ten times larger toroidal field than the radial component, i.e., with a rather low pitch angle.

A lot of models, but mostly axisymmetric, were published during that period. Most of the models were investigating different aspects of the turbulence, looking also for 
parameters where non-axisymmetric solutions could appear. Another application was the effect of radial flows on the dynamo properties driven by the Maxwell stress of the dynamo generated magnetic field. Models including a wind were investigated, especially to explain vertical fields above the disk - notably Brandenburg et al. (1993); Elstner et al. (1995). Both models used different assumptions about the halo diffusivity and the wind structure. In the model of Brandenburg et al. (1993), diamagnetism was included, and because of the positive turbulence gradient, the pumping was not an escape velocity but an inward transport term. In Elstner et al. (1995), a passive halo with low conductivity was assumed. These models already showed a fast growth time below 0.1 Gyr. All these models could in principle explain the main features of the observed magnetic fields in nearby spiral galaxies. But the connection between optical and magnetic spirals was still unclear.

With the advent of the new century, full-blown 3D models became feasible, and one could now attack the spiral structure. Simple artificial velocity models adopted from density-wave theory were now taken into account. One spectacular observational result was the anti-correlation of optical and magnetic arms, found by Beck \& Hoernes (1996). A turbulent dynamo could reproduce such a behaviour due to the different properties of the turbulence in the spiral arms and in the inter-arm regions - see Rohde \& Elstner (1998), Shukurov (1998) and Rohde et al. (1999).

More elaborated models used the velocity from numerical galaxy models computed with SPH or sticky-particle methods (Elstner et al. 2000). Models for spiral galaxies produced no inter-arm fields by the large-scale spiral motion alone. Only with a variation of the turbulence, one could have inter-arm fields. The situation was different for bar galaxies (Otmianowska-Mazur et al. 2002). In these simulations, one could observe interarm magnetic fields in the outer spiral arm, and the field lagged behind the optical arm (Moss et al. 1998, cf.). More recently, Beck et al. (2005) and Moss et al. (2007) conducted models for barred galaxies with stationary flows.

Another problem was raised in the context of the so-called catastrophic quenching: Because of helicity conservation, in the ideal MHD case, the $\alpha$ term will be quenched (with the square-root of the magnetic Reynolds number) if there is no additional transport of magnetic helicity in the box. Under the assumption of a galactic wind or fountain flow, these problems may disappear. But a too strong wind would also result in a suppression of the dynamo (Sur et al. 2007). Recent investigations have shown that already the galactic shear flow can suppress the catastrophic quenching of the dynamo (Brandenburg 2009; Käpylä et al. 2008).

The recent box model of the turbulent ISM driven by multiple clustered supernova explosions (Gressel et al. 2008), for the first time demonstrated the presence of a dynamo process by direct numerical simulations. It turned out that the turbulence coefficients derived from the SOCA models are in good agreement with the new results from the direct simulations. In a similar way, the box simulations of Hanasz et al. (2004) have shown an exponential growth of the magnetic field. Their simulations, however, do not resolve the supernova remnant in itself. Instead, the explosion site only serves as a source for the released cosmic ray energy. Due to the fast diffusion of cosmic rays, the pressure redistribution is fast enough to allow for similarly short growth times of several $100 \mathrm{Myr}$. The role of a magnetic instability of the Parker type could be essential for this process, too.

In general, the effect of magnetic instabilities in releasing kinetic energy should be considered as a possible mechanism for magnetic field amplification in galaxies - especially in regions with low star formation activity. The primary candidate of this type is the magneto-rotational instability (MRI), which has a growth time on the order of 
the galactic rotation frequency and thus can become efficient on very short timescales. With todays computational resources, global models of the MRI are already within reach (Dziourkevitch et al. 2004; Nishikori et al. 2006). However, these models still have to assume quite strong initial fields to properly resolve the fastest growing modes of the instability.

\section{The pitch angle problem}

When it comes to the explanation of the observed magnetic fields in spiral galaxies, there is one main reason for favouring the dynamo hypothesis over the primordial field hypothesis, namely the existence of fields - of equipartition strength - with a large pitch angle. If the field amplification were mainly due to the shearing motion of the differential rotation, the field would have to be wound up several times to explain its observed strength. This, however, would lead to an amplification of the toroidal field only, resulting in a configuration with an intrinsically small pitch angle.

For the dynamo scenario, which furthermore implies exponential growth, we additionally have an amplification of the poloidal field. The pitch angle $p$ of the growing field in the kinematic regime of the $\alpha \Omega$ dynamo can be estimated from the dimensionless dynamo numbers $C_{\alpha}$ and $C_{\Omega}$ (for a definition see below) via

$$
p=\arctan \left(\sqrt{\frac{C_{\alpha}}{C_{\Omega}}}\right) .
$$

This leads to values between $5^{\circ}$ and $30^{\circ}$, for reasonable values of $C_{\alpha}=5-10$ and $C_{\Omega}=25-$ 800. For a given rotational velocity, the angular frequency is defined by the turnover radius, i.e., the point where the rotation curve becomes flat. Small turnover radii lead to rather large $C_{\Omega}$ and thus to low pitch angles. In the saturated regime, the situation becomes worse - at least for the conventional $\alpha \Omega$ dynamo. This is because $\alpha$ will then be reduced by non-linear effects and the pitch angle fades.

Direct simulations of the ISM turbulence show a possible way out of this dilemma, namely by another saturation process, which we will demonstrate in the following section. Moreover, the action of the magneto-rotational instability may be a reasonable candidate for the explanation of large pitch angles as they are observed in maps of polarised synchrotron emission for instance in the ringed galaxy NGC 4736 (Chyży \& Buta 2008).

\section{Global models with turbulence coefficients from direct simulations}

Global simulations of interstellar turbulence remain out of reach, at least if one is interested in resolving the relevant scales. Therefore, we apply a hybrid approach, where we consider the mean induction equation

$$
\frac{\partial \vec{B}}{\partial t}=\operatorname{curl}\left(\vec{u} \times \vec{B}+\alpha \circ \vec{B}-\eta_{\mathrm{T}} \circ \operatorname{curl} \vec{B}\right)
$$

for the mean magnetic field $\vec{B}$ and apply closure parameters obtained from direct simulations. The background rotation serves as the main part of the mean flow

$$
u_{\varphi}=r \Omega_{0}\left(1+\left(\frac{r}{r_{\Omega}}\right)^{2}\right)^{-1 / 2}
$$



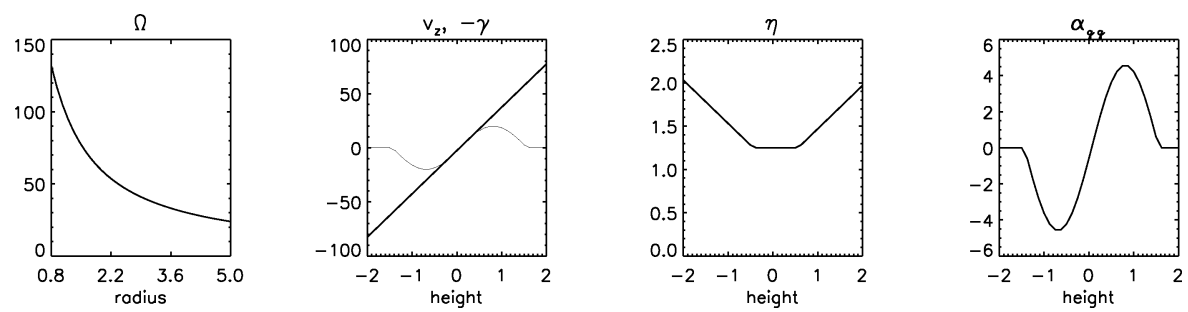

Figure 1. Radial dependence of $\Omega$ and vertical profiles of the fountain flow and turbulent transport coefficients.

and is supplemented by a vertical wind $u_{z}=u_{0} z$ which is a result of the densitystratified turbulence driven by SNe (cf. Fig. 2 in Gressel et al. 2009). The test-field method, furthermore, yields an $\alpha$ tensor of the form

$$
\alpha=\alpha_{0}\left(\begin{array}{ccc}
\alpha_{r r} & -\gamma_{z} & 0 \\
\gamma_{z} & \alpha_{\varphi \varphi} & 0 \\
0 & 0 & \alpha_{z z}
\end{array}\right)
$$

where the dependence of these quantities on the height $z$ is modelled according to the profiles in Gressel et al. (2008).

For a realistic global galaxy model one further needs the radial dependence of the relevant effects. The radial profiles of the rotational frequency, SN rate, gravitational potential and density are in principle known. By means of a parameter study, we try to obtain the $\alpha$ effect from our box simulations, accordingly. From these, we find a dependence of the diagonal terms of the $\alpha$ tensor on rotation, namely $\alpha_{\varphi \varphi} \propto \Omega^{0.5}$. This is weaker than expected from SOCA theory, which predicts a scaling linear with $\Omega$ and only a minor rotational quenching. Consistent with SOCA, we find the turbulent diamagnetism $\gamma_{z}$ to be nearly independent of $\Omega$. For the turbulent diffusivity $\eta_{\mathrm{t}}$, we apply only horizontal components which also vary according to $\Omega^{0.5}$. The dependence on the supernova rate is given in Gressel et al. (2009).

After proper normalisation with a length of $l=1 \mathrm{kpc}$ and a diffusion time $t_{\mathrm{D}}=l^{2} / \eta_{0}$ with $\eta_{0}=1 \mathrm{kpc}^{2} \mathrm{Gyr}^{-1}$, the dynamo is characterised by the Reynolds numbers

$$
C_{\alpha}=\alpha_{0} l / \eta_{0}, \quad C_{\Omega}=\Omega_{0} l^{2} / \eta_{0} \quad \text { and } \quad C_{w}=u_{0} l / \eta_{0} .
$$

We assume a disk of radius $R=5 \mathrm{kpc}$ and with a height of $H=2 \mathrm{kpc}$, according to our box dimension in the direct simulations. We approximate the profiles of the turbulence coefficients by smooth functions (see Fig. 1). The vertical dependence of the $\alpha$ tensor can simply be reproduced by a sine function

$$
\alpha(z) \propto \sin \left(z \pi / \mathrm{h}_{\alpha}\right)
$$

with a scale height $h_{\alpha}=1.5 \mathrm{kpc}$. The mean vertical velocity is linearly growing with height. Only the diagonal terms of the $\alpha$ tensor have a radial dependence proportional to $\Omega$, whereas the other terms are constant. We, furthermore, use a simple $\alpha$ quenching

$$
\alpha=\alpha_{0} /\left(1+B^{2} / B_{\text {eq }}^{2}\right)
$$

for the full tensor, i.e., including the diamagnetic term $\gamma_{z}$.

In our first run, we neglect turbulent diamagnetism and mean vertical velocity, as it is done in the classical $\alpha \Omega$ dynamo. In this model, we find a growth time of $700 \mathrm{Myr}$, a field strength for the toroidal component $B_{\varphi}=20 B_{\text {eq }}$ and the radial component $B_{r}=0.6 B_{\text {eq }}$. If we neglect only the fountain flow, the field decays. For the real model, we find a 

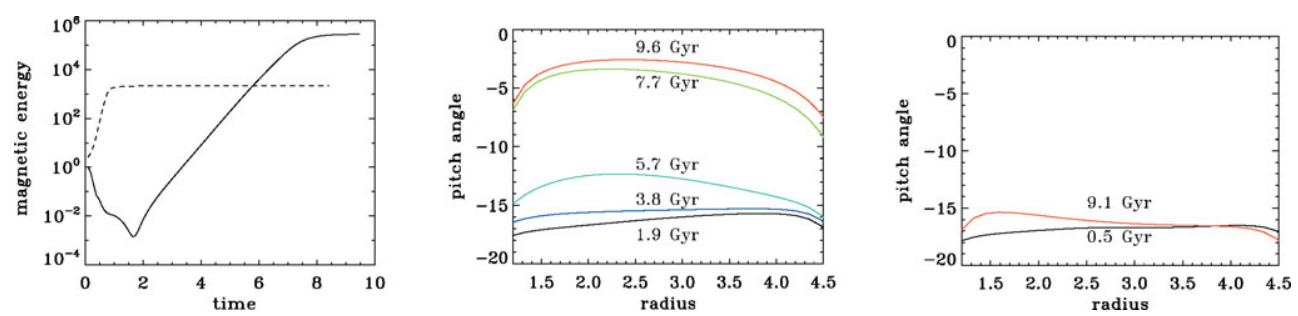

Figure 2. Left: Time evolution of magnetic energy for model A, with wind (dashed) and model F, without wind and pumping (solid). Middle: Time evolution from 1.9 Gyr until 9.6 Gyr of the radial pitch angle distribution for model F. Right: Pitch angle for model A at $0.5 \mathrm{Gyr}$ and 9.1 Gyr.
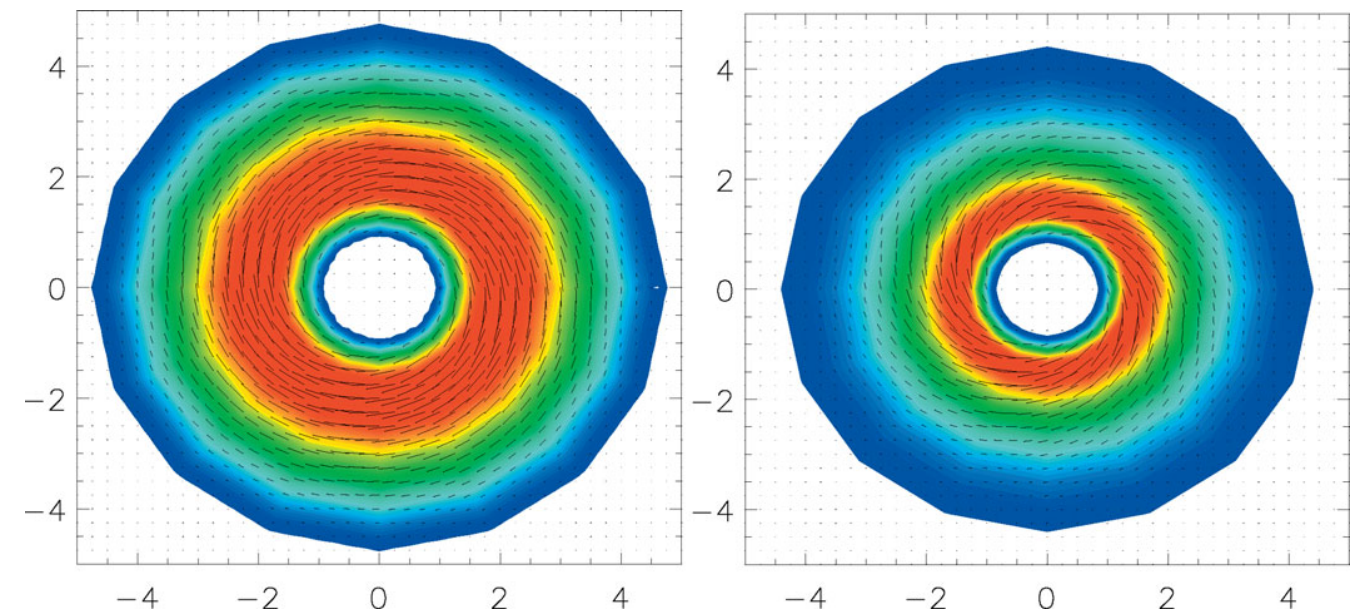

Figure 3. Magnetic vectors as observed in polarised emission (neglecting Faraday rotation) for the final fields in model F (left) and model A (right)

growth rate of $250 \mathrm{Myr}$ - similar to the box simulation. The field now saturates earlier with $B_{\varphi}=1.1 B_{\text {eq }}$ and $B_{r}=0.3 B_{\text {eq }}$.

The final state is not determined by the usual $\alpha$ quenching in this case. Instead, a combination of fountain flow and quenching of the diamagnetic term leads to the saturation of the dynamo. This means, the dynamo stops growing because of field losses by the fountain flow. As a nice consequence of this non-linear feedback, the pitch angle remains large in the saturated regime (see Fig. 2).

Moreover, in Fig. 3 we present directions of the magnetic field resulting from polarised emission of synchrotron radiation, where we assumed a scale height of $500 \mathrm{pc}$ for the relativistic electron distribution.

In Table 1 , the pitch angle is given for a choice of different models. Comparing $p=$ $\arctan \left(\sqrt{C_{\alpha} / C_{\Omega}}\right)$ with the initial pitch angle $p_{0}$ during the kinematic growth phase, we find reasonable agreement. The final value $p_{\text {final }}$ in the saturated state for the first three models is similar to the value during the kinematic growth phase.

In order to have a higher pitch angle for our rotation law with $r_{\Omega}=1 \mathrm{kpc}$, we need larger values of $C_{\alpha}$ and, consequently, a larger vertical outflow. Otherwise the $\alpha$ quenching would reduce the final pitch angle - as is the case in model E. This increase of the mean outflow velocity with increasing $\mathrm{SN}$ rate is indeed observed in the box simulations 
Table 1. Pitch angles for different dynamo models

\begin{tabular}{lrrrrrrrc}
\hline & $C_{\alpha}$ & $C_{\Omega}$ & $p$ & $p_{0}$ & $p_{\text {final }}$ & $v_{\text {rot }}$ & $u_{0}$ & $\gamma / \alpha_{\varphi \varphi}$ \\
\hline $\mathrm{A}$ & 5 & 100 & -13 & -16 & -16 & 100 & 20 & 2 \\
$\mathrm{~B}$ & 5 & 200 & -9 & -10 & -10 & 200 & 20 & 2 \\
$\mathrm{C}$ & 5 & 50 & -18 & -17 & -15 & 50 & 20 & 2 \\
$\mathrm{D}$ & 25 & 100 & -27 & -28 & -18 & 100 & 20 & 2 \\
$\mathrm{E}$ & 25 & 100 & -27 & -28 & -27 & 100 & 40 & 2 \\
$\mathrm{~F}$ & 5 & 100 & -13 & -16 & -3 & 100 & 0 & 0 \\
\hline
\end{tabular}

(cf. Gressel et al. 2009). For model F, one can see a dramatic reduction of the pitch angle due to the missing mean vertical velocity and diamagnetic pumping.

\section{Magneto-Rotational Instability}

Galaxies are known to be unstable with respect to the magneto-rotational instability. This instability, discovered by Velikhov (1959) and Chandrasekhar (1960), has meanwhile been studied in great detail in the astrophysical context (see e.g. Balbus \& Hawley 1991; Kitchatinov \& Mazur 1997). Sellwood \& Balbus (1999) pointed to the possibility of turbulence generation by the MRI in regions of low star formation activity. Piontek \& Ostriker (2007) recently simulated the MRI in a vertically stratified two-phase ISM box model.

Kitchatinov \& Rüdiger (2004) investigated the stability of global galactic disks. For a disk threaded by a weak vertical field, they estimated a field strength of $10^{-25} \mathrm{G}$ for the onset of the instability. Accordingly, the MRI should already be present during the galaxy formation process - and therefore a good candidate for the seed field of a turbulent dynamo. The most unstable mode in their idealised galactic disk model is of quadrupolar type. A very interesting result is the large pitch angle of $45^{\circ}$ for the fastest growing mode. For weak fields, the unstable modes have a small spatial scale but with the property of a uniform large pitch angle. This leads to a smooth map of magnetic vectors for the polarised radio emission, but the Faraday rotation measure will be small for these types of magnetic fields.

The instability, moreover, has a very short growth time proportional to the rotation time. A proof for a large scale dynamo driven by the MRI in the sense, that a small initial field can be amplified over several magnitudes has not been given yet. This is because it is numerical difficult to resolve the small spatial scales for the unstable modes connected to the weak initial field. Nevertheless, there are hints that the process is possible. Especially the turbulence excited by MRI in a stratified disk gives a non-vanishing $\alpha$ effect, but with negative sign in the northern hemisphere (Arlt \& Rüdiger 2001; Brandenburg \& Sokoloff 2002).

Since we are interested in configurations with large pitch angles, we here consider global models of a density-stratified galactic disk with a uniform vertical field. Tests with toroidal fields did not produce a comparable radial field component. For simplicity, we assumed an isothermal disk where the rotation and vertical density stratification is supported by the gravitational potential. Without magnetic fields the configuration was tested to be in a stationary equilibrium over $10 \mathrm{Gyr}$ with only small velocity fluctuations below $0.01 \mathrm{~km} \mathrm{~s}^{-1}$, which are slowly decaying with time.

The applied density profile is

$$
\rho=\rho_{0} \exp \left(\frac{-4 z^{2}}{z^{2}+3 h_{\rho}^{2}}\right)
$$



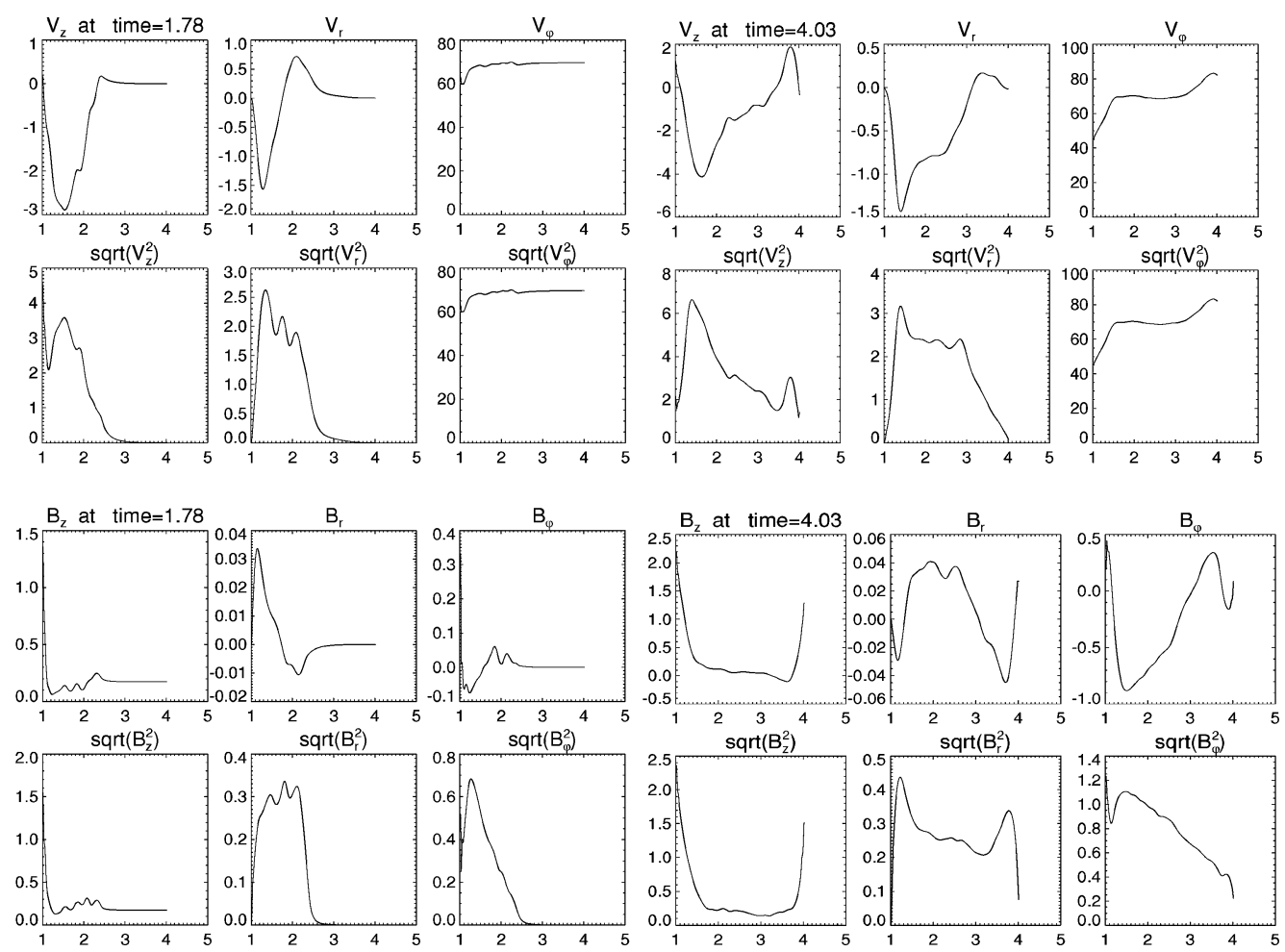

Figure 4. Radial profiles of $\varphi, z$ averaged velocity components in $\left[\mathrm{km} \mathrm{s}^{-1}\right]$ (first row), rms-velocity components in $\left[\mathrm{km} \mathrm{s}^{-1}\right]$ (second row), $\varphi, z$-averaged magnetic field components in $[\mu \mathrm{G}]$ (third row) and rms magnetic field components in $[\mu \mathrm{G}]$ (fourth row) at 1.8 Gyr.

with the scale height $h_{\rho}=0.3 \mathrm{kpc}$ and $\rho_{0}=10^{-24} \mathrm{gcm}^{-3}$. Note that the density minimum is about $0.02 \rho_{0}$. The rotation curve is, furthermore, defined by (3.2) with the turnover radius $r_{\Omega}=0.35 \mathrm{kpc}$ and $\Omega_{0}=200 \mathrm{Gyr}^{-1}$. The inner radius of our disk model is $r_{\text {in }}=$ $1 \mathrm{kpc}$ and the outer radius $r_{\mathrm{out}}=4 \mathrm{kpc}$. The vertical extent is $\pm 5 \mathrm{kpc}$. Within $\pm 1 \mathrm{kpc}$, we use a constant grid spacing with 256 grid points and a non-uniform grid for the outer regions with 128 points at each side. This means, the inner disk has a resolution of about $7 \mathrm{pc}$ and for the outer disk we yield about $80 \mathrm{pc}$. This is consistent with a density variation of a factor 100, in order to get a comparable growth of the unstable modes for a uniform magnetic field strength within our restricted resolution.

The radial boundary is reflecting and the vertical boundary is reflecting for the velocity only, while the tangential component of the magnetic field and the gradient of the vertical component are set to zero. Under these conditions, the vertical flux is conserved but a development of horizontal flux is allowed. Applying a weak and uniform vertical magnetic field of $0.1 \mu \mathrm{G}$, we find an exponential growth of the unstable mode after a transient relaxation phase of about $200 \mathrm{Myr}$. The field starts to grow at the inner radial boundary, where $\Omega$ has a maximum. As the field grows further, it spreads out radially. At the same time, the Maxwell stress causes an inflow in the inner part of the magnetised region. At the outer magnetic region, we observe an outflow of mass, which is a consequence of angular momentum conservation.

Because of our closed boundaries, the density increases strongly at the inner boundary and moderately at the outer boundary. We see a weak change of the rotation curve at the 
boundaries, compensating the developing pressure gradients. This is probably an artefact of the closed boundaries. Tests with larger radial domains have shown that the change of the rotation curve is restricted to the boundary region.

Finally, the field growth saturates. At the moment it is unclear whether the saturation is caused by the non-linear feedback of the developed turbulence. The reduction of the pitch angle could in fact be a hint that the system reaches the diffusive limit of the instability, where we know from linear analysis that the unstable mode, in this regime, is mainly toroidal. On the other hand, we see a large-scale meridional circulation in the disk. Moreover, our model disk has a limited mass reservoir, which could be another reason for the early saturation with rather low magnetic field strength. As long as the toroidal and radial field strength are below the field strength of the initial vertical field, the field parallel to the disk grows with a time of $0.05 \mathrm{Gyr}$; later we observe growth times of $0.8 \mathrm{Gyr}$ until the saturation sets in. It should be noted that the growth-time of a possible MRI-driven dynamo has not to be as fast as the growth time of the instability.

In Fig. 4, we plot radial profiles which have been averaged along the vertical and azimuthal direction for $-5 \mathrm{kpc}<\mathrm{z}<0 \mathrm{kpc}$ and $0<\varphi<2 \pi$. The values shown are the components of the velocity, magnetic field and their corresponding rms values. A largescale meridional circulation develops with an amplitude of roughly 0.5 of the rms value ( $\simeq 6 \mathrm{~km} \mathrm{~s}^{-1}$ for the vertical component and $\simeq 3 \mathrm{~km} \mathrm{~s}^{-1}$ for the radial component).

The mean magnetic field along the $\mathrm{z}$ and $\varphi$ direction are of the same order as the rms values of the fields. Only the mean radial field is by an order of magnitude smaller than the rms field. We find $B_{r}^{\mathrm{rms}} / B_{\varphi}^{\mathrm{rms}}=0.3$ and for the mean-field $B_{r} / B_{\varphi}=0.05-0.1$, corresponding to pitch angles of $17^{\circ}$ and $3^{\circ}-6^{\circ}$, respectively. In contrast, the pitch angle in the polarisation map varies from $10^{\circ}$ to $20^{\circ}$ if the boundaries are excluded $(1.3 \mathrm{kpc}<\mathrm{r}<3.6 \mathrm{kpc})$.

Initially, at time $1.8 \mathrm{Gyr}$, the pitch angle varies from $20^{\circ}$ to $40^{\circ}$ for $1.3 \mathrm{kpc}<\mathrm{r}<2 \mathrm{kpc}$. In the outer region the field is still too weak, and we do not observe any polarised emission. Fig. 5 shows a slice through the magnetic field at $z=-0.8 \mathrm{kpc}$, the vertically averaged field along the lower disk half $(-5 \mathrm{kpc}<\mathrm{z}<0 \mathrm{kpc})$ and the magnetic direction of polarised emission (neglecting Faraday rotation). Varying the scale height height of the relativistic electron distribution from $0.5 \mathrm{kpc}$ to $2.0 \mathrm{kpc}$ gave no significant differences. Note the smooth polarisation map for an incoherent magnetic field (i.e., with many reversals along the vertical direction). The pitch angle is here much larger than the pitch angle for the averaged field.

\section{Summary}

New direct simulations of supernova-driven turbulence in a Cartesian box have shown the existence of the turbulent dynamo in a galactic environment. We find the resulting balance of the turbulent diamagnetism with the mean vertical outflow to be crucial for the operation of the dynamo. Using the turbulence of the direct simulations for the determination of the transport coefficients, one can show that the fast dynamo growth time of a few hundred million years is due to the fast transport by the mean vertical fountain flow and the downward pumping of the turbulent diamagnetism. This is in contrast to the slow conventional $\alpha \Omega$ dynamo with growth times of the order of $1 \mathrm{Gyr}$, where only diffusion contributes to a vertical field transport.

We have shown the possibility of an alternative saturation process other than the simple quenching of the diagonal $\alpha$ term. In the case of quenching via the transport mechanisms, the pitch angle of the final saturated field will not deplete. But still, there 

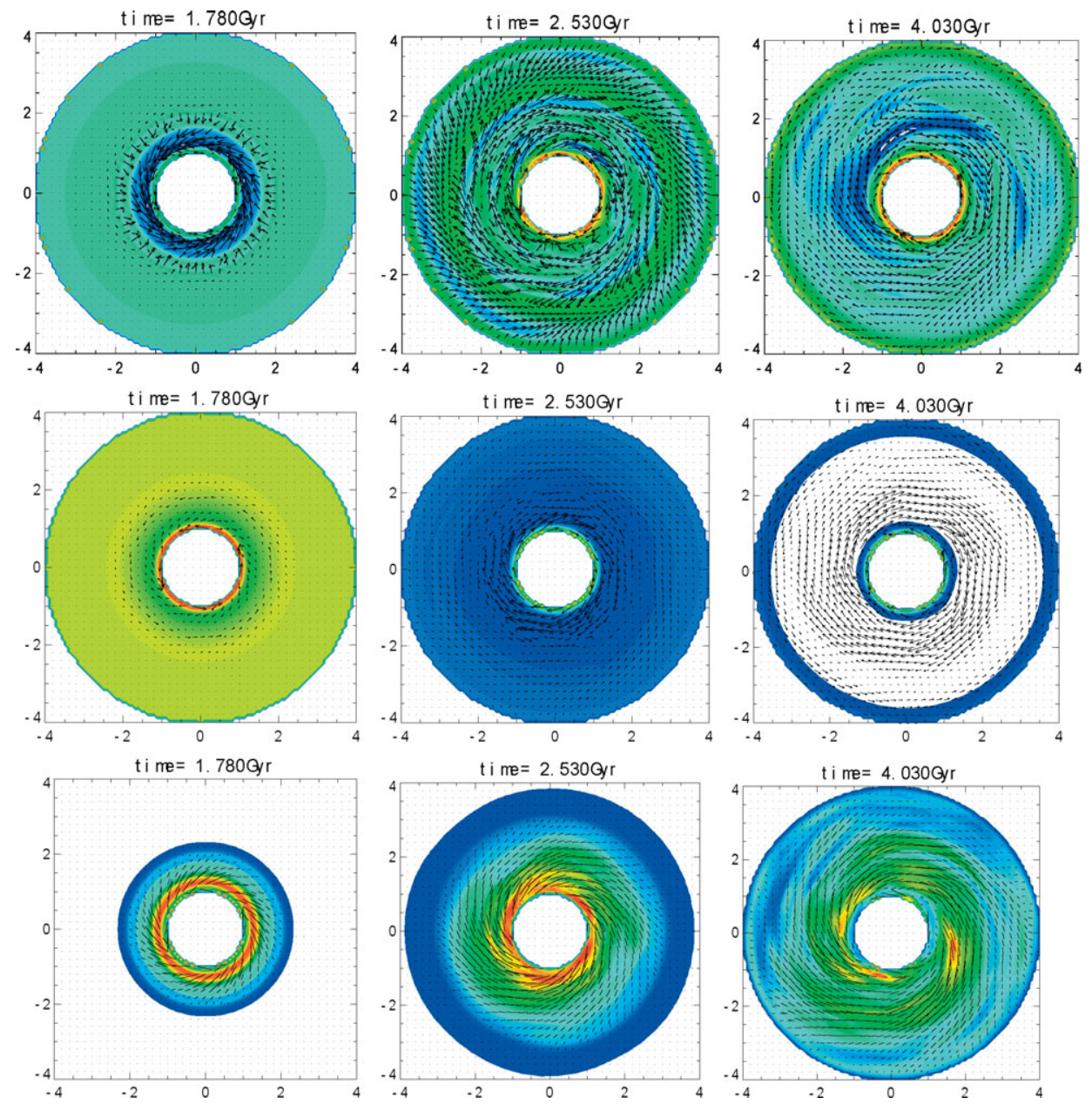

Figure 5. Magnetic field slices at $z=-0.8 \mathrm{kpc}$ (first row) and averaged field over one disk half (second row) with colour coded z component. Magnetic field direction from polarised emission with colour coded total intensity (third row).

are cases of very strong differential rotation where it may be difficult to get observed pitch angles of up to $40^{\circ}$ with actual SN-driven dynamo models.

More detailed investigations of the ISM model must be performed. For instance, cosmic rays could further improve the situation. Also the effects of strong magnetic fields have to be considered, as magnetic instabilities could contribute to further amplification processes. The magneto-rotational instability would be a good candidate - not only as a generator for the seed field, but also for the generation of magnetic fields with large pitch angles. It is not clear, however, whether an MRI driven dynamo can generate enough vertical flux through the disk in order to get solutions with large enough pitch angles, which are known from the linear unstable modes. But at least under a given vertical flux, the MRI leads to incoherent magnetic fields, which appear in the polarised synchrotron emission (neglecting Faraday rotation) as smooth spiral fields. The mean-field generated in such models has a smaller pitch angle than the polarised emission vectors, so a significant contribution from a small scale field enhances the pitch angle in the polarisation 
map. Future RM synthesis observations would be very helpful for the understanding of magnetic fields in external galaxies.

\section{Acknowledgements}

We thank N. Dziourkevitch and A. Bonanno for helpful discussions and support concerning the MRI computations. We are also grateful to U. Ziegler for supplying the NIRVANA code.

\section{References}

Arlt, R. \& Rüdiger, G. 2001 A\&A 374, 1035

Balbus, S. A. \& Hawley, J. F. 1991, ApJ 376, 214

Beck, R. \& Hoernes, P. 1996, Nature 379, 47

Beck, R., Brandenburg, A., Moss, D., Shukurov, A., \& Sokoloff, D. 1996, ARAA 34, 155

Beck, R., Fletcher, A., Shukurov, A., Snodin, A., Sokoloff, D. D., Ehle, M., Moss, D., \& Shoutenkov, V. 2005, A\&\&A 444, 739

Beck, R. 2009, this proceedings

Brandenburg, A., Donner, K. J., Moss, D., Shukurov, A., Sokoloff, D. D., \& Tuominen, I. 1993, $A \& A 271,36$.

Brandenburg, A. \& Sokoloff, D. D. 2002, GApFD 96, 319

Brandenburg, A. 2009, this proceedings

Chandrasekhar, S. 1960, Proc. Nat. Acad. Sci. 46, 253

Chyży, K. T. \& Buta, R. J. 2008, ApJ 677, L17

Donner, K.J.\& Brandenburg, A. 1990, A\&A 240, 289

Dziourkevitch, N., Elstner, D., \& Rüdiger, G. 2004, A\& $A$ 423, L29

Elstner, D., Meinel, R., \& Beck, R. 1992, A\&SAS 94, 587

Elstner, D., Golla, G., Rüdiger, G., \& Wielebinski, R. 1995, A\&\&A 297, 77

Elstner, D., Otmianowska-Mazur, K., von Linden, S., \& Urbanik, M. 2000, A\&A A 357, 129

Ferrière, K. \& Schmitt, D. 2000, A\&SA 358, 125

Ferrière, K. 1998, A\&SA 335, 488

Fröhlich, H.E. \& Schultz, M. 1996, A\&A 311, 451

Gressel, O., Elstner, D., Ziegler, U., \& Rüdiger, G. 2008, A $64 A$ 486, L35

Gressel, O., Ziegler, U., Elstner, D., \& Rüdiger, G. 2009, this proceedings

Hanasz, M., Kowal, G., Otmianowska-Mazur, \& K. Lesch, H. 2004, ApJ 605, L33

Kaisig, M., Rüdiger, G., \& Yorke, H. W. 1993, A\&A 274, 757

Käpylä, P. J., Korpi, M. J., \& Brandenburg, A. 2008, A\& $A$ 491, 353

Kitchatinov, L.L. \& Mazur, M.V. 1997, A\&A 324, 821

Kitchatinov, L.L. \& Rüdiger, G. 2004, A\&A 424, 565

Moss, D., Korpi, M., Rautiainen, P., \& Salo H. 1998, A\&A 329, 895

Moss, D., Snodin, A., Englmaier, P., Shukurov, A., Beck, R., \& Sokoloff, D. 2007, A\&A 465, 157

Nishikori, H., Machida, M., \& Matsumoto, R. 2006, ApJ 641, 862

Otmianowska-Mazur, K., Elstner, D., Soida, M., \& Urbanik, M. 2002, A\&̋A 384, 48

Parker, E. N. 1971, ApJ 164, 491

Piontek, R A. \& Ostriker, E. C. 2007, ApJ 663, 183

Rohde, R. \& Elstner, D. 1998, A\&A 333, 27

Rohde, R., Beck, R., \& Elstner, D. 1999, A\& A 350, 423

Rüdiger, G. \& Kitchatinov, L.L. 1993, A\&\&A 269, 581

Sellwood, J.A. \& Balbus, S.A. 1999, ApJ 511, 660

Shukurov, A. 1998, MNRAS 299, L21

Stix, M. 1975, A\&GA 42, 85-89.

Sur, S., Shukurov, A., \& Subramanian, K. 2007, MNRAS 377, 874.

Velikhov, E.P. 1959, Sov. Phys. JETP 9, 995

White, M. 1978, AN 299, 209

Ziegler, U. 1996, A\&A 313, 448 


\section{Discussion}

De Gouveia Dal Pino: We have recently published results of global 3D simulations of galactic fountain evolution and we have found that for the typical SN rates of our galaxy, the fountain flows reach a maximum rate of $\approx 4 \mathrm{kpc}$ and they attain a "steady" state (with the amount of matter going up becoming equal to the amount going down and returning to the disk) in about $150 \mathrm{Myr}$ (Melioli et al. 2008, MNRAS). So my question is, how do the dynamo parameters depend on these properties, i.e. the SN rate and height of the fountain flow?

ELSTNER: We investigated the dependence on the SN-rate for smaller value than the galactic value and found a square-root dependence. The relaxation time for the system to develop a stationary state is between 100-200 Myr.

FLETCHER: Have you compared your mean-field models to the recently published models developed by Shukurov, Subramanian, Sur and Brandenburg involving the outward transport of small-scale magnetic helicity by a galactic wind?

Elstner: Yes we compared that. But due to the turbulent diamagnetism, we have a broader dynamo regime.

REICH: Do you think you can simulate the galactic magnetic field in terms of your simulations, or are there features left not fitting well?

ELSTNER: In order to get the galactic magnetic field, you probably have to include some evolution information like detailed star formation mass. Also initial condition and the environment field may be necessary. The main question remains, why our galaxy with it's reversals should be exceptional.

Otmianowska-Mazur: Do you get the $\alpha$ and $\eta$ coefficients from EMF? Do you think that your process is connected with the Parker instability?

ELSTNER: The turbulence coefficients are determined by the test-field method. The process is probably not connected with the Parker instability because the turbulence parameters are founded already in the hydro regime

SOKOLOFF: It is very pity that because of shortage of time the speaker omitted to remark that the galactic dynamo models suggested in 1990th by Shukurov et al., Brandenburg et al., Moss et al., Beck et al. were much more successful in reporting observations than the model by Ferrière and they resulted in something very similar to what was presented in this impressive detailed simulations.

ELSTNER: Yes I did not mention all models based on SOCA estimates for the mean field simulations in the 90th. They were already successful, because SOCA is a good prediction, which we now learn from the direct simulations. 


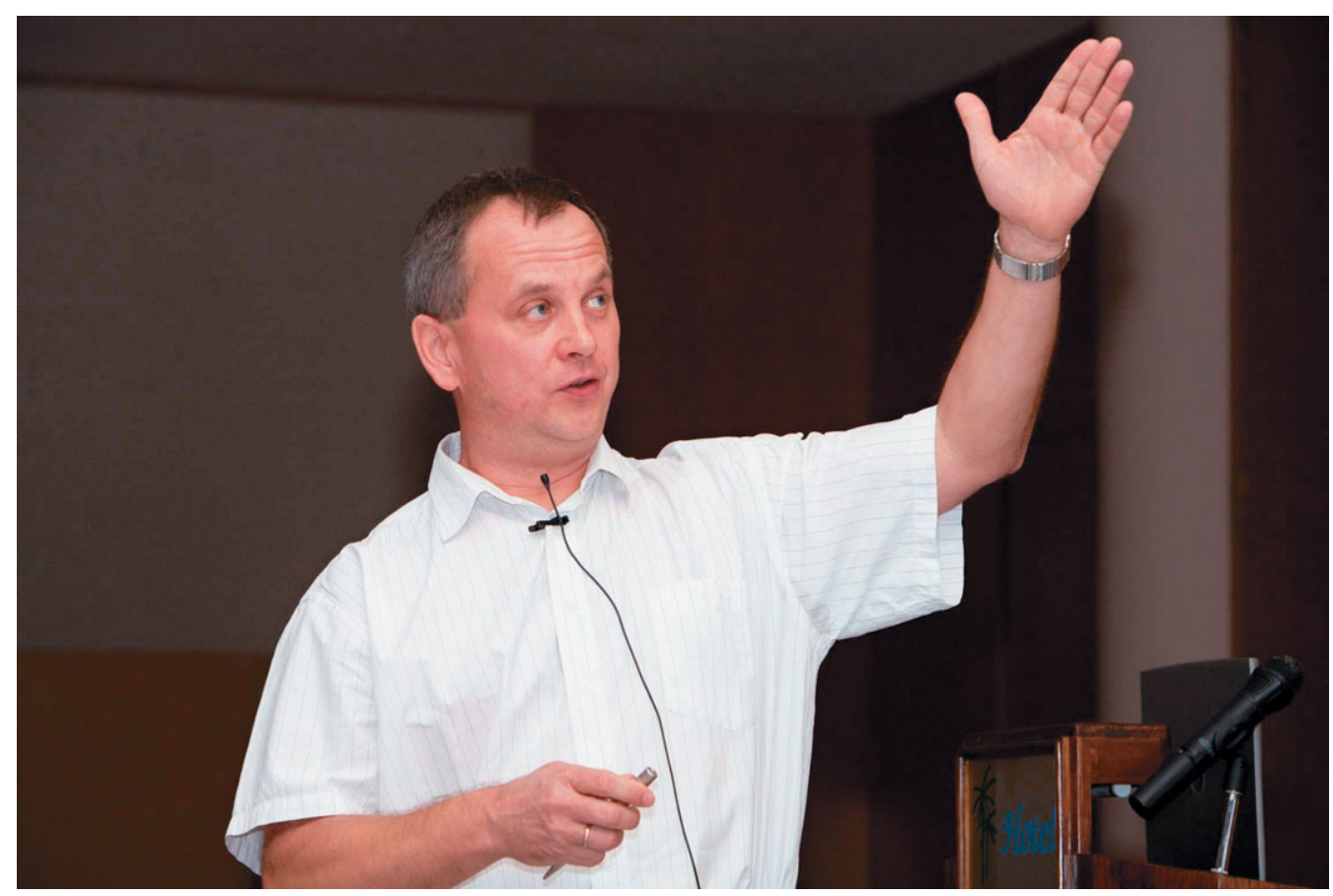

Michal Hanasz

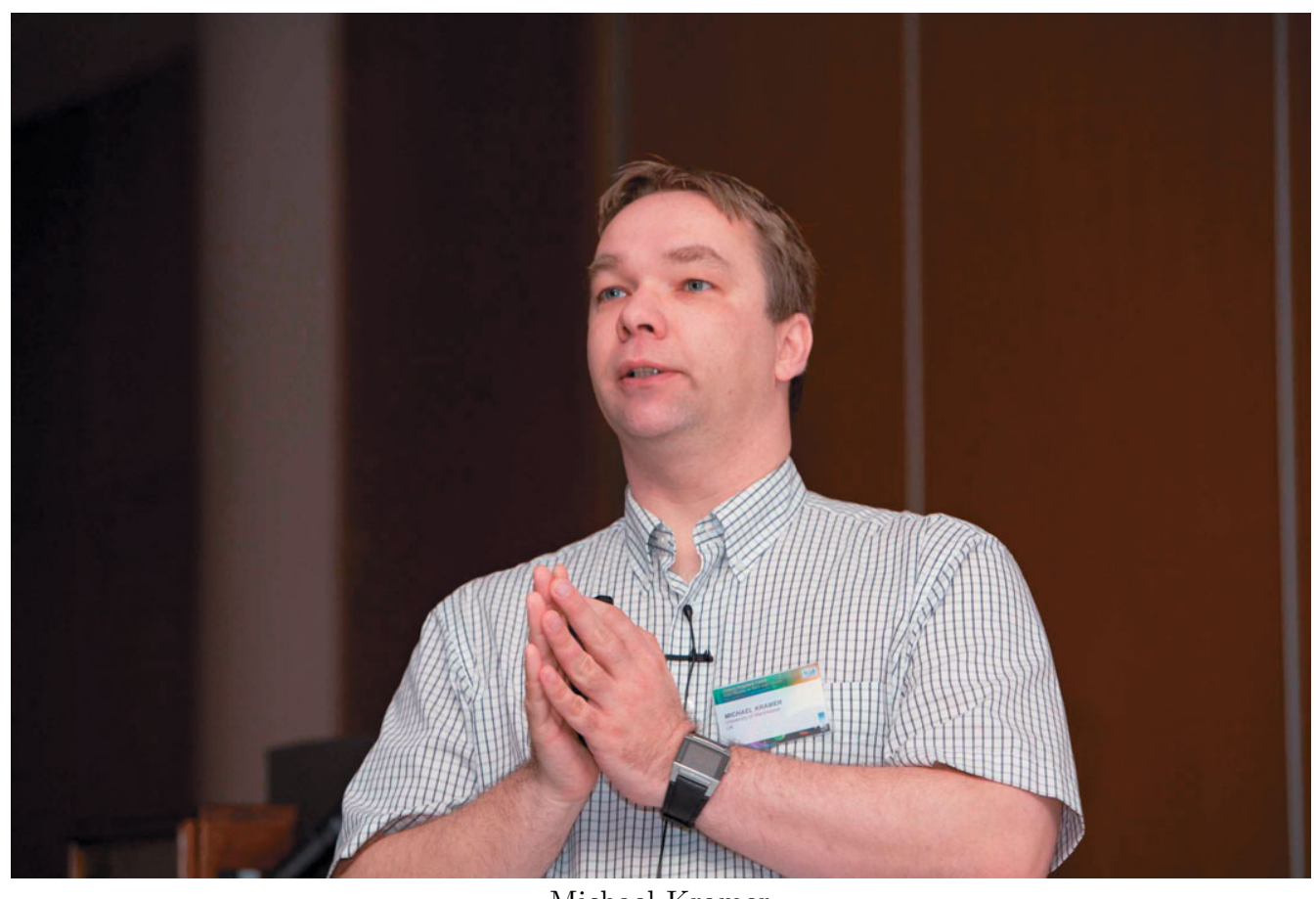

Michael Kramer 